\title{
Prefrontal activation in suicide attempters during decision making with emotional feedback
}

\author{
Adrián Alacreu-Crespo (1) ${ }^{1,2}$, Emilie Olié $\mathbb{0}^{1,2}$, Emmanuelle Le Bars ${ }^{3,4}$, Fabienne Cyprien², Jérémy Deverdun ${ }^{4}$ and \\ Philippe Courtet ${ }^{1,2}$
}

\begin{abstract}
Emotional feedback, such as faces showing emotions, can influence decision making. Decision making and emotional face processing, mainly mediated by the prefrontal and cingulate cortices, are impaired in suicide attempters. Here, we used functional MRI (fMRI) to study prefrontal activation in suicide attempters during a modified version of the lowa Gambling Task (IGT) that included emotional face feedback. We randomly distributed the 116 euthymic women $(n=$ 45 suicide attempters, $n=41$ affective controls with history of depression without suicide attempt, and $n=30$ healthy controls) included in the study in three emotional IGT groups: concordant (safe and risky choices followed by happy and angry faces, respectively), discordant (safe and risky choices followed by angry and happy faces, respectively), and neutral condition (safe and risky choices followed by neutral faces). Considering the two IGT phases (ambiguous and risky), we then analyzed five regions of interest during the risky vs. safe choices: orbitofrontal (OFC), anterior cingulate (ACC), ventrolateral (VLPFC), medial (MPFC) and dorsal prefrontal (DPFC) cortices. We found: (1) impaired decision making and increased DPFC and OFC activation in suicide attempters vs. controls in the discordant condition during the risky phase; (2) reduced VLPFC activation in suicide attempters in the concordant condition during the ambiguous phase; and (3) decreased OFC, ACC and DPFC activation in both control groups in the concordant condition during the ambiguous phase. Suicide attempters showed prefrontal alterations during reward-learning decision making with emotional feedback. Suicide attempters may guide their decisions to avoid social negative feedback despite the expected outcome.
\end{abstract}

\section{Introduction}

Suicidal acts may be viewed as the outcome of dysfunctional decision making. In a phenomenological qualitative analysis that included eight suicide attempters, Bergman et al. ${ }^{1}$ reported a precarious decision-making state about their own life and destiny. Several studies that used the Iowa Gambling Task (IGT) ${ }^{2}$ found that decision making is impaired in subjects with history of suicidal acts, but not suicidal ideation ${ }^{3-10}$. IGT is a decisionmaking task with monetary feedback after each choice.

Correspondence: Adrián Alacreu-Crespo (adrian.alacreu@uv.es)

${ }^{1}$ Department of Emergency Psychiatry and Acute Care, CHU Montpellier, Montpellier, France

${ }^{2}$ PSNREC, Univ Montpellier, INSERM, CHU de Montpellier, Montpellier, France Full list of author information is available at the end of the article

These authors contributed equally: Adrián Alacreu-Crespo and Emilie Olié
This feedback should allow participants to learn which choices are safe (i.e., larger wins than losses) and risky (i.e., larger losses than wins) for earning money during 100 trials $^{11}$. IGT is a decision-making task that involves value-based learning and presents two degrees of uncertainty: an ambiguous phase when subjects cannot estimate the outcome (first 40 trials), and a risky phase when subjects can better estimate the possible outcome (last 60 trials $)^{12,13}$. Compared with patients without history of suicide attempt, decision-making is impaired in the risky phase of the IGT in suicide attempters ${ }^{4,7-10}$.

Some neuroimaging studies have been performed to identify the neural bases of decision making as a suicidal vulnerability trait. Jollant et al. ${ }^{14}$ performed functional magnetic resonance imaging (fMRI) during the IGT and found that orbitofrontal cortex (OFC) activation was

\section{(c) The Author(s) 2020}

(c) Open Access This article is licensed under a Creative Commons Attribution 4.0 International License, which permits use, sharing, adaptation, distribution and reproduction cc) in any medium or format, as long as you give appropriate credit to the original author(s) and the source, provide a link to the Creative Commons license, and indicate if changes were made. The images or other third party material in this article are included in the article's Creative Commons license, unless indicated otherwise in a credit line to the material. If material is not included in the article's Creative Commons license and your intended use is not permitted by statutory regulation or exceeds the permitted use, you will need to obtain permission directly from the copyright holder. To view a copy of this license, visit http://creativecommons.org/licenses/by/4.0/. 
decreased during risky choices (relative to safe choices) in euthymic suicide attempters compared with patients without suicide history. In a replication study, Olié et al. ${ }^{15}$ showed that dorsal prefrontal cortex (DPFC) activation was decreased during the risky compared with safe choices in suicide attempters. They also reported increased OFC, DPFC and anterior cingulate cortex (ACC) activation during monetary wins relative to losses in suicide attempters compared with non-attempters. Furthermore, differences in OFC and DPFC activation have been observed in relatives and non-relatives of suicide completers during the IGT risky choices compared with safe choices ${ }^{16}$.

During value-based decision making, OFC role is to assess the risk level depending on the reward value ${ }^{17}$. Conversely, DPFC is implicated in the cognitive control of actions and in the integration of complex rules ${ }^{18,19}$. Therefore, it has been hypothesized that both emotional and motivational processes are involved in the decisionmaking impairment observed in suicide attempters ${ }^{20}$.

Every day people are confronted with a variety of emotional/social cues and they must interpret their underlying meaning in function of the context. According to the somatic marker hypothesis ${ }^{21}$, emotional feedback and the related physiological activations act as signals that might guide future choices. Some studies demonstrated that emotional feedback can bias decision-making processes, even in the presence of monetary feedback ${ }^{22-24}$. More concretely, in the IGT, healthy people with congruent monetary/emotional feedback (happy faces) chose more safe decks than risky decks compared to subjects with incongruent feedbacks (fearful faces) or no feedback $^{25}$. In addition, suicide attempters have difficulties in interpreting and integrating emotional/social signals. For instance, when viewing angry faces relative to neutral faces, activation of OFC and ventrolateral prefrontal cortex (VLPFC) is increased and DPFC activation is decreased in suicide attempters compared with patients without history of suicidal acts. ${ }^{15,26}$. Moreover, ACC activation is increased in suicide attempters when viewing happy faces relative to neutral faces ${ }^{15,26}$. This suggests that suicide attempters might be more sensitive to negative than positive emotional cues. This hypothesis leads to the question of whether suicide attempters would benefit differently from emotional feedback during decisionmaking processes compared with non-attempters.

To address this question, we used fMRI to compare brain activation in patients with and without lifetime history of suicide attempts and in healthy controls (reference group) during a modified version of the IGT. To avoid gender bias and the effect of acute depression, we recruited only euthymic women. This modified version of the IGT included emotional feedback using angry, happy and neutral faces that were shown between the card choice and the monetary feedback. The emotional feedback given by these images might modulate the decisionmaking performances. Based on previous research, we hypothesized that suicide attempters would not have the same behavioral benefits from emotional feedback than affective and healthy controls, and that this would be mirrored also by different OFC and DPFC activation profiles.

\section{Methods and materials \\ Participants}

This study enrolled 116 euthymic women, aged between 19 and 54 years (mean \pm SEM $=36.96 \pm 0.82$ ). This sample was used in a previous fMRI study on brain processing of social rejection ${ }^{27}$. Participants were included if they met the inclusion criteria indicated below, and then were screened in person by a psychiatrist. All women were Caucasian, right-handed (Edinburgh Handedness Inventory $)^{28}$, and euthymic at the time of the fMRI scan, as indicated by their Hamilton Depression Rating Scale $(\text { HDRS })^{29}$ and Young Mania Rating Scale $\left(\right.$ YMRS) ${ }^{30}$ scores (both $<7$ ). Exclusion criteria were: lifetime history of severe head trauma, central nervous system disorder, schizophrenia, history of alcohol or drug abuse or dependence within the past 12 months, pregnancy, and contraindications to MRI.

Participants were divided in three groups: patients with past history of major depressive episode and suicide attempt (suicide attempters $=\mathrm{SA} ; n=45$ ), patients with history of depressive episode without suicidal behavior (affective controls $=\mathrm{AC} ; \mathrm{n}=41$ ), and subjects without psychiatric history (healthy controls $=\mathrm{HC} ; n=30$ ). A suicide attempt was defined as a self-damaging act carried out with certain intention to die. It is different from selfmutilation, the use of substances, or non-compliance with medical treatments ${ }^{31}$. Diagnoses were made according to the DSM-IV criteria using the Mini-International Neuropsychiatric Interview 5.0 (MINI) ${ }^{32}$.

Patients were recruited among the outpatients of the Department of Emergency Psychiatry \& Post-Acute Care of Montpellier Academic Hospital (France). HCs were recruited through advertisement and from a list of volunteers in the Montpellier Academic Hospital database.

The Montpellier University Hospital Ethics Committee (CPP Sud Mediterranée IV) approved the study. A signed informed written consent was obtained from all participants. All participants received $100 €$ for their participation in the study.

\section{Clinical assessment}

In the week preceding the fMRI, the following data were collected; (1) sociodemographic characteristics and medication intake using ad-hoc structured interviews, 
(2) depressive symptomatology using the Beck Depression Inventory $(\mathrm{BDI})^{33}$, (3) state and trait anxiety using the State-Trait Anxiety Inventory (STAI) ${ }^{34}$, (4) impulsivity using the Barrat Impulsiveness Scale (BIS-10) ${ }^{35}$, (5) verbal intelligence quotient (IQ) using the French version of the National Adult Reading Task (NART) ${ }^{36}$, (6) and past history of childhood trauma using the Childhood Trauma Questionnaire (CTQ) $)^{37}$.

\section{Modified IGT}

The IGT models real-life decision making because participants are initially unaware of the underlying contingencies. Specifically, two decks are long-term advantageous ("safe decks") because wins are low but losses are lower, leading to a net gain. The other two decks are disadvantageous ("risky decks") because wins are high but losses higher, leading to a net loss. Each choice leads to a gain or a loss of a variable money amount. The goal of the game is to win as much money as possible. Individuals learn from experience to avoid the risky decks. An IGT modified for fMRI studies ${ }^{2,38}$ was used. Four decks of cards (two safe and two risky) were presented on a screen. Participants were prompted to pick a card from the deck of their choice ("Pick a card"). After each choice, a positive or negative amount of virtual money (win or loss) was shown for $2 \mathrm{~s}$. The IG index was the number of choices from the safe decks minus those from the risky decks for all 100 choices. The number of choices from the safe decks minus those from risky decks for trials 1-40 and from trials 41-100 represented the IG ambiguous (i.e., decision making under ambiguous uncertainty) and IG risk (i.e., decision making under risk uncertainty), respectively. After the task completion, the explicit understanding of the task contingencies was rated from 0 (none) to 2 (completely understood).

In our modified version of the IGT, emotional feedback (i.e., pictures of angry, happy or neutral faces previously used by the team ${ }^{15}$ ) was added between the choice (card picking) and the monetary feedback to create three different feedback conditions: concordant (safe and risky choices followed by happy and angry faces, respectively), discordant (safe and risky choices followed by angry and happy faces, respectively), and neutral condition (safe and risky choices followed by neutral faces) independently of the monetary gains or losses. Figure 1 summarizes the procedure.

Within the three groups, participants were randomly distributed as follows: SA group $(n=14$ in the neutral, $n=16$ in the concordant, and $n=15$ in the discordant condition), AC group ( $n=13$ in the neutral, $n=15$ in the concordant, and $n=13$ in the discordant condition), and HC group $(n=10$ in the neutral, $n=11$ in the concordant, and $n=9$ in the discordant condition). All participants were task-naïve.

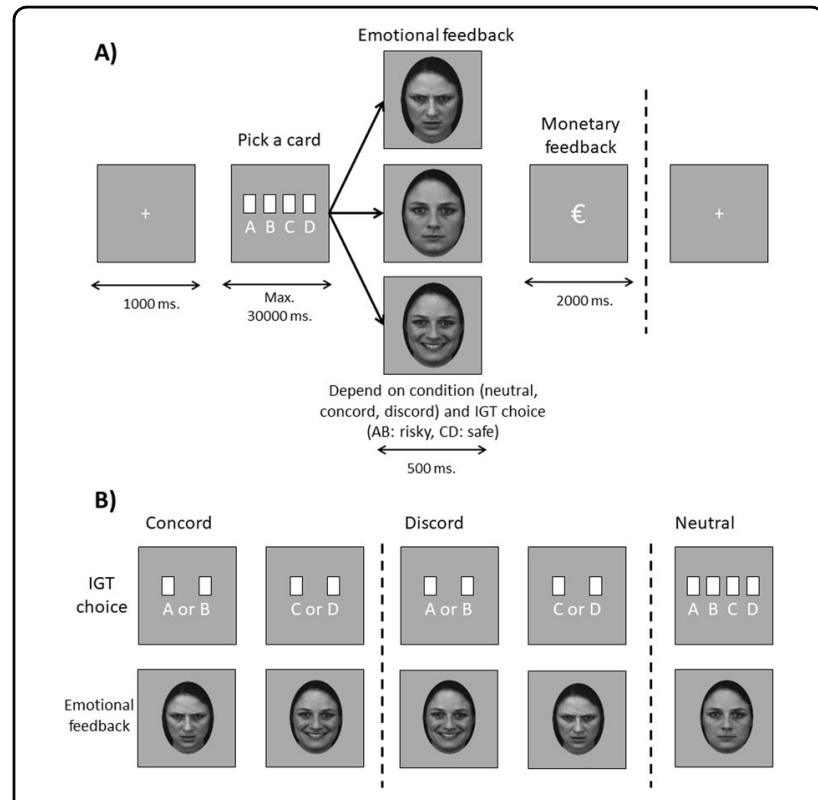

Fig. 1 Modified version of the lowa Gambling Task (IGT). a fMRI during the modified IGT with different (neutral, concordant, discordant) emotional feedback relative to the choice. $\mathbf{b}$ Summary of the different emotional feedback conditions ( $\mathbf{a}$, $\mathbf{b}$ : risky decks; $\mathbf{c}$, d: safe decks of cards). Note: Concord concordant, Discord discordant.

\section{Image acquisition}

fMRI images were acquired at the Neuroradiology Department - I2FH (Academic Hospital of Montpellier) using a $1.5 \mathrm{~T}$ whole-body MRI system (MAGNETON AVANTO, Siemens, Erlangen, Germany) equipped with a standard 12-channel receive-only head coil. Image acquisition during the IGT task was performed using gradient echo-echo planar imaging (GE-EPI) with the following parameters: $\mathrm{TR}=3 \mathrm{~s}, \mathrm{TE}=40 \mathrm{~ms}, \mathrm{FOV}=$ $220 \mathrm{~mm}$, 42 slices, voxel size $=3.75 \times 3.75 \times 3.3 \mathrm{~mm}$, flip angle $90^{\circ}, 403$ volumes.

An additional 3D magnetization-prepared, rapid acquisition gradient echo (MP-RAGE) sequence was also obtained for each participant with the following parameters: $\mathrm{TR}=2100 \mathrm{~ms}, \mathrm{TE}=4.1 \mathrm{~ms}, \mathrm{IR}=1100 \mathrm{~ms}, 15^{\circ}$ flip angle, $\mathrm{PAT}=2$, aligned with the corpus callosum, voxelsize $0.98 \times 0.98 \times 1 \mathrm{~mm}, 160$ transversal slices.

\section{Statistical analysis \\ fMRI data analysis}

Data were analyzed using SPM12 (Wellcome Department of Imaging Neuroscience, London, UK) and Matlab R2019a (Mathworks, Inc., Natick, MA). The first four volumes of each fMRI run were discarded due to the time needed to launch the IGT synchronized with fMRI acquisition. Then, the first 40 trials corresponded to the Ambiguous and the others to the Risky phase of the IGT. GE-EPI data were re-oriented to the anterior commissure, 
slice-time corrected, realigned to the first volume, coregistered, spatially normalized (based on T1) using the DARTEL algorithm, and smoothed with an 8-mm FWHM Gaussian filter.

Contrast images were computed for risky vs. safe choices for each participant using a first-level general linear model. Realignment parameters were added in the regressor to remove specific activation of head movements and a high-pass filter (cut-off $=128 \mathrm{~s}$ ) was used to remove non-physiological slow signal shifts. A secondlevel analysis was then performed using a factorial design to evaluate the interaction between Group (SA, AC, HC) and Condition (Neutral, Concordant, Discordant) separately for the two IGT phases (Ambiguous and Risky). For the voxel wise analysis, significance threshold was set at $p<0.001$ (uncorrected), with $k \geq 10$ voxels.

Specific regions of interest (ROI) were chosen on the basis of an a priori hypothesis concerning the specific anatomical brain regions that could be associated with decision making, as done in Olié et al. ${ }^{15}$. The ROIs were anatomically defined by Anatomical Automatic Labeling (AAL), using masks provided by the Wake Forest University PickAtlas software (http://fmri.wfubmc.edu). OFC was defined as the orbital parts of the inferior frontal gyrus, middle frontal gyrus and medial frontal gyrus (including the medial and lateral parts) (corresponding to the Brodmann areas (BA) 11/47), VLPFC as the opercular part of the inferior frontal gyrus (BA 44/45), MPFC as the medial part of the superior frontal gyrus (BA 10), and ACC (BA 24/32), and DPFC as the middle frontal gyrus (BA 8/9/46) (Supplementary Fig. 1).

\section{Clinical and behavioral data analysis}

The variable normality was checked with the Kolmogorov-Smirnov test and homogeneity of variances with the Levene test; non-normal variables were log transformed. Preliminary ANOVAs or Student's $t$ tests (for continuous variables) and chi-square or Fisher's tests (categorical variables) were performed with Group (SA $v s$ $\mathrm{AC} v s \mathrm{HC})$ as factor to test differences in sociodemographic and clinical variables. For variables showing significant differences in the three groups, simple contrast post-hoc tests were performed with False Discovery Rate (FDR) correction. Variables found to be significant with the post-hoc test were used as covariates in the analysis of the behavioral responses.

Then, two-way ANCOVAs for Group (SA vs AC vs HC) and Condition (Neutral vs Concordant vs Discordant) were performed using IG ambiguous and IG risky as dependent variables. The mean reaction time during the choices was calculated for each IGT phase, and the same analysis was done with reaction times as dependent variables. Simple contrast post-hoc tests were performed with FDR correction.
The alpha significance level was fixed at 0.05 , and the threshold for significant trends at 0.07 . All statistical analyses were performed with SPSS 20.0.

\section{Results \\ Demographic and clinical variables}

Sociodemographic variables, NART score and IGT explicit understanding were not significantly different in the three groups (all $p>.05$ ) (Table 1). As expected, the STAI state and trait scores were lower in the HC group than in the two patient groups. Moreover, HCs tended to report less frequently childhood emotional abuse and neglect compared with the two patient groups.

In the two patient groups, bipolar disorder was more frequent in the SA than AC group $(p<0.012)$, while the other clinical variables were comparable (all $p>0.05$ ).

Therefore, lifetime bipolar disorder was used as covariate in the behavioral analyses.

\section{Behavioral results \\ Reaction times for choices}

In the IGT ambiguous phase, there were significant Group differences $\left(F_{2,106}=4.41, p<0.014, \eta^{2}=.08,1-\beta=\right.$ 0.76). Reaction times were faster in the AC group than in the SA $(p<0.029)$ and HC groups $(p<0.029$; mean \pm SEM: $\quad \mathrm{SA}=831.25 \pm 40.99 \mathrm{~ms}, \quad \mathrm{AC}=708.38 \pm 32.43 \mathrm{~ms}$, $\mathrm{HC}=824.54 \pm 32.99 \mathrm{~ms}$ ). Moreover, reaction times were longer in participants who received neutral feedback than in participants who received concordant emotional feedback (mean \pm SEM: Neutral $=860.83 \pm 34.56 \mathrm{~ms}$, Concordant $=725.95 \pm 32.68 \mathrm{~ms}$, Discordant $=777.38 \pm$ $34.97 \mathrm{~ms}$ ) (Condition factor: $F_{2,106}=3.86, p<0.024, \eta^{2}=$ 0.07, power $=0.70)$ (Fig. 2a).

In the IGT risky phase, there was a significant trend for the Condition factor $\left(F_{2,106}=2.80, p<0.065, \eta^{2}=0.05\right.$, power $=0.55)$. However, post-hoc comparisons did not show any significant difference (mean \pm SEM: Concordant $=$ $686.36 \pm 26.76 \mathrm{~ms}$., Discordant $=707.96 \pm 28.91 \mathrm{~ms}$., Neutral $=775.78 \pm 28.57$ ms.) (Fig. 2a).

\section{IGT performances}

In the IGT ambiguous phase, no Group or Condition effect and no Group $\times$ Feedback interaction was observed ( $p$ values $>0.05$ ) (Fig. 2b).

In the IGT risky phase, there was a significant Group $\times$ Condition interaction $\left(F_{4,106}=2.75, p<0.031, \eta^{2}=0.09\right.$, $1-\beta=0.76)$. Post-hoc comparisons showed that the IG from the risky blocks was higher in the $\mathrm{HC}$ than $\mathrm{AC}(p<$ $0.023)$ and SA $(p<0.007)$ groups in the discordant feedback condition. Moreover, within the SA group, the IG from the risky blocks tended to be lower in patients in the discordant feedback condition than in the other two conditions ( $p<0.061$ for both) (Fig. 2b). 
Table 1 Comparison of sociodemographic and clinical variables in healthy controls, affective controls and suicide attempters.

\begin{tabular}{|c|c|c|c|c|}
\hline & Healthy controls $(n=30)$ & Affective controls $(n=41)$ & Suicide attempters $(n=45)$ & $p$ values and post-hoc \\
\hline \multicolumn{5}{|l|}{ Sociodemographic data } \\
\hline Age & $37.45(1.54)$ & $36.16(1.29)$ & $37.56(1.29)$ & $p=0.698$ \\
\hline Years of education & $14.63(.37)$ & $14.49(.31)$ & $13.85(.31)$ & $p=0.208$ \\
\hline \multicolumn{5}{|l|}{ Clinical characteristics } \\
\hline Age at first thymic episode & - & $24.41(1.27)$ & $23.00(1.29)$ & $p=0.436$ \\
\hline Number of depressive episodes & - & $3.24(1.24)$ & $6.28(1.26)$ & $p=0.090$ \\
\hline Number of manic episodes & - & $1.83(1.22)$ & $2.72(1.40)$ & $p=0.633$ \\
\hline BDI score & $0.50(0.19)$ & $4.44(0.75)$ & $4.73(0.62)$ & $p=0.001, A C, S A>H C$ \\
\hline HAMD score & $1.33(0.30)$ & $3.93(0.36)$ & $3.84(0.39)$ & $p=0.001, A C, S A>H C$ \\
\hline YMRS score & $0.03(0.03)$ & $0.44(0.21)$ & $0.53(0.29)$ & $p=0.342$ \\
\hline STAl state score & $27.23(1.05)$ & $36.34(1.64)$ & $35.64(1.53)$ & $p=0.001, A C, S A>H C$ \\
\hline STAI trait score & $31.13(1.26)$ & $44.10(1.49)$ & $46.69(1.58)$ & $p=0.001, A C, S A>H C$ \\
\hline BIS-10 total score & $42.90(1.96)$ & $47.02(2.12)$ & $50.13(2.11)$ & $p=0.069$ \\
\hline IGT comprehension & $0.92(0.16)$ & $0.63(0.13)$ & $0.64(0.12)$ & $p=0.264$ \\
\hline NART score & $21.40(0.75)$ & $21.90(0.64)$ & $22.22(0.61)$ & $p=0.696$ \\
\hline \multicolumn{5}{|l|}{ Lifetime psychiatric comorbidities } \\
\hline Bipolar disorder, $n(\%)$ & - & $12(29.3)$ & $25(55.6)$ & $p=0.012$ \\
\hline Eating disorder, $n(\%)$ & - & $4(9.8)$ & $7(15.6)$ & $p=0.421$ \\
\hline Anxiety disorder, $n(\%)$ & - & $20(48.8)$ & $27(60.0)$ & $p=0.297$ \\
\hline PTSD, $n(\%)$ & - & $2(4.9)$ & $5(11.1)$ & $p=0.437$ \\
\hline Alcohol/Substances abuse, $n$ (\%) & - & $5(12.2)$ & $10(22.2)$ & $p=0.221$ \\
\hline \multicolumn{5}{|l|}{ Suicidal history } \\
\hline Age at first suicide attempt & - & - & $24.51(3.28)$ & - \\
\hline Number of suicide attempts & - & - & $1.29(.39)$ & - \\
\hline Violent suicide attempt, Yes $n(\%)$ & - & - & $2(4.4)$ & - \\
\hline Severe suicide attempt, Yes $n(\%)$ & - & - & $9(20.0)$ & - \\
\hline \multicolumn{5}{|l|}{ Medication } \\
\hline Benzodiazepines, Yes $n(\%)$ & - & $1(2.4)$ & $7(15.6)$ & $p=0.060$ \\
\hline Antidepressants, Yes $n$ (\%) & - & $9(22.0)$ & $7(15.6)$ & $p=0.447$ \\
\hline Antiepileptics, Yes $n(\%)$ & - & $2(4.9)$ & $8(17.8)$ & $p=0.093$ \\
\hline Antipsychotics, Yes $n(\%)$ & - & $1(2.4)$ & $7(15.6)$ & $p=0.060$ \\
\hline Lithium, Yes $n(\%)$ & - & $3(7.3)$ & $4(8.9)$ & $p=0.790$ \\
\hline \multicolumn{5}{|l|}{ Childhood trauma moderate/severe } \\
\hline CTQ physical abuse, Yes $n(\%)$ & $1(3.3)$ & $3(7.3)$ & $3(6.7)$ & $p=0.765$ \\
\hline СTQ physical neglect, Yes $n(\%)$ & $2(6.7)$ & $7(17.1)$ & $9(20.0)$ & $p=0.278$ \\
\hline CTQ emotional abuse, Yes $n(\%)$ & $3(10.0)$ & $14(34.1)$ & $19(42.2)$ & $p=0.011 \mathrm{AC}, \mathrm{SA}>\mathrm{HC}$ \\
\hline CTQ emotional neglect, Yes $n(\%)$ & $4(13.3)$ & $11(26.8)$ & $22(48.9)$ & $p=.004 \mathrm{SA}>\mathrm{HC}$ \\
\hline CTQ sexual abuse, Yes $n(\%)$ & $1(3.3)$ & $6(14.6)$ & $9(20.0)$ & $p=0.120$ \\
\hline
\end{tabular}

Data are shown as means \pm SEM and frequencies.

$M D D$ major depressive disorder, BDI beck depression inventory, HAMD Hamilton Depression Rating Scale, YMRS Young Mania Rating Scale, STAI State-Trait Anxiety Inventory, BIS-10 Barrat Impulsiveness Scale, IGT lowa Gambling Task, NART National Adult Reading Task, PTSD Post-traumatic stress disorder, CTQ Childhood Trauma Questionnaire, $H C$ healthy controls, $A C$ affective controls, $S A$ suicide attempters.

\section{Functional MRI}

Table 2 summarizes the fMRI results during the IGT ambiguous and risky phases. Supplementary Fig. 2 shows the maps of brain activation without ROI masking during the ambiguous and risky phases. Supplementary Fig. 3 shows the comparison of ROI beta values in the three groups ( $\mathrm{HC}, \mathrm{AC}$, and $\mathrm{SA})$ during the risky vs. safe choice contrast.
Risky vs. Safe choices during the ambiguous IGT phase

In the concordant vs. neutral feedback condition contrast, bilateral VLPFC activation was reduced in the SA group (Left: $p<0.001, k=47$; Right: $p<0.001, k=26$ ); bilateral activation of ACC (Left: $p<0.001, k=25$; Right: $p<0.001, k=26$ ), OFC (Left: $p<0.001, k=10$; Right: $p<$ $0.001, k=21$ ) and DPFC (Left: $p<0.001, k=19$; Right: $p<0.001, k=15)$ was decreased in the $\mathrm{AC}$ group; and 
A)

RT Ambigous phase

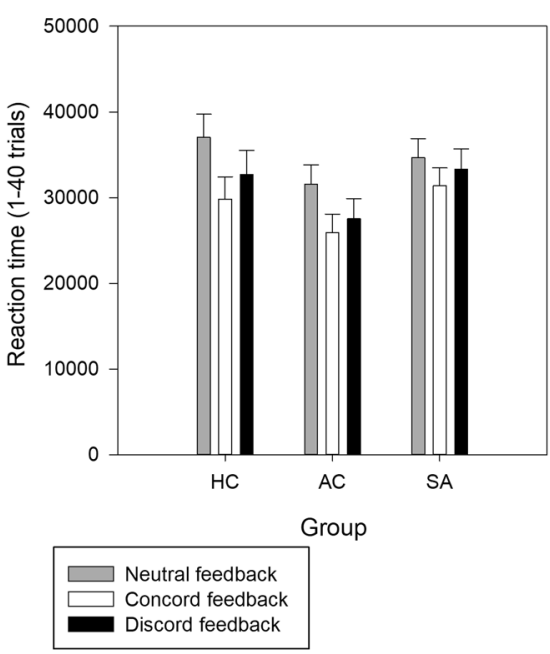

B) IG Ambigous phase

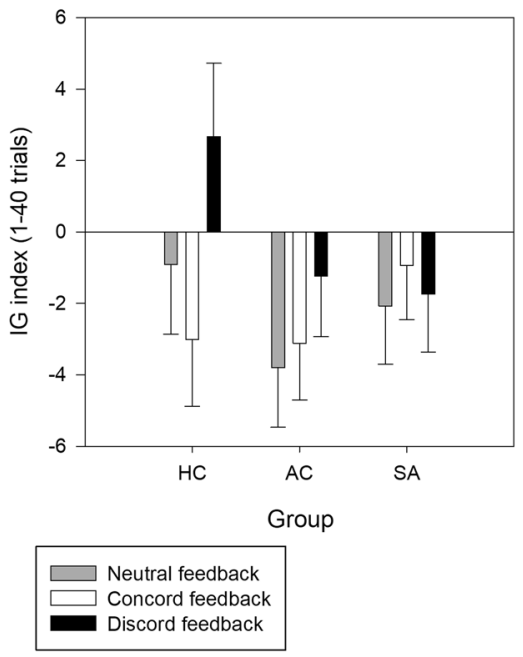

RT Risky phase

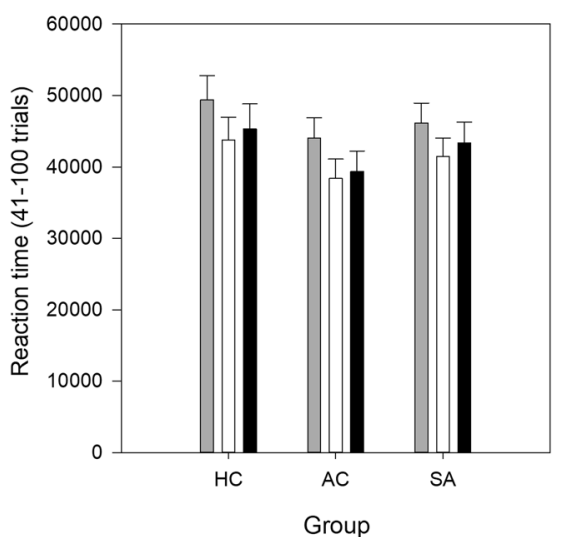

IG Risky phase

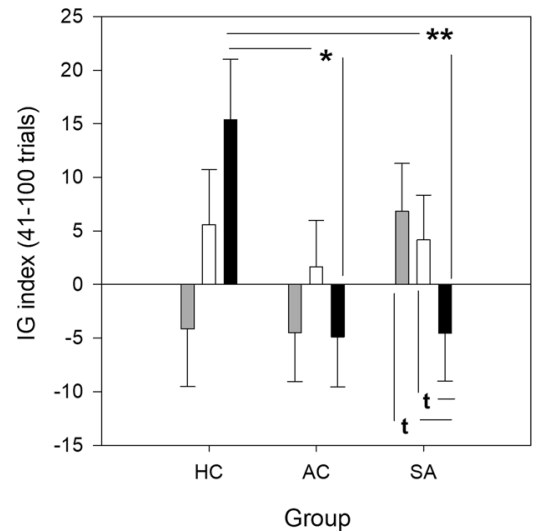

Fig. 2 Behavioral results of ambigous and risky phases in the lowa Gambling Task. a Total reaction time (RT) in the three groups and in the three emotional feedback conditions during the ambiguous (i.e., decision making under ambiguous uncertainty) and risky (i.e., decision making under risk uncertainty) phases of the lowa Gambling Task. Data are the mean \pm SEM of the total reaction time in seconds for the ambiguous and risky phases. b Performance (IG index) in the three groups and in the three emotional feedback conditions during the ambiguous (i.e., decision making under ambiguous uncertainty) and risky (i.e., decision making under risk uncertainty) phases of the lowa Gambling Task. Group (SA vs AC vs HC) by condition (Neutral vs Concordant vs Discordant) interaction. Data are the mean \pm SEM of the IG score for the ambiguous and risky phases. Note: ${ }^{* *}=p$ $<0.01,{ }^{*}=p<0.05,{ }^{\mathrm{t}}=p<.07 ; \mathrm{HC}$ healthy controls, AC affective controls, SA suicide attempters.

activation of the left ACC $(p<0.001, k=18)$ and right OFC $(p<0.001, k=69)$ was reduced in the HC group (Fig. 3a).

In the discordant vs. neutral feedback condition contrast, activation was decreased in the right DPFC $(p<0.001, k=99)$ and left VLPFC $(p<0.001, k=16)$ in the $\mathrm{HC}$ group.

\section{Risky vs. Safe choices during the risky IGT phase}

In the concordant vs. neutral feedback condition contrast, activation of the right ACC $(p<0.001, k=41)$ and left DPFC $(p<0.001, k=27)$ and MPFC $(p<0.001, k=14)$ was reduced in the $\mathrm{AC}$ group.

In the discordant vs. neutral feedback condition contrast, activation was increased in the right DPFC 
Table 2 Significant group $\mathrm{x}$ emotion comparisons, voxel $p<0.001, k \geq 10$.

\begin{tabular}{|c|c|c|c|c|}
\hline IGT ambiguous phase & $\mathrm{K}_{\mathrm{E}}$ & $\mathrm{T}$ & $(x, y, z)$ & Selected ROI \\
\hline \multicolumn{5}{|l|}{ Neutral > Concordant } \\
\hline \multirow[t]{2}{*}{ Suicide attempters } & 47 & 4.11 & $(-50,34,10)$ & VLPFC (L) \\
\hline & 26 & 4.04 & $(56,12,4)$ & VLPFC (R) \\
\hline \multirow[t]{8}{*}{ Affective controls } & 26 & 4.09 & $(6,32,24)$ & $A C C(R)$ \\
\hline & 25 & 4.11 & $(-4,20,32)$ & $A C C(L)$ \\
\hline & 21 & 4.16 & $(36,18,-6)$ & OFC (R) \\
\hline & 19 & 4.03 & $(-22,30,48)$ & DPFC $(L)$ \\
\hline & 15 & 3.57 & $(50,26,32)$ & DPFC (R) \\
\hline & 14 & 3.75 & $(6,60,-2)$ & OFC (R) \\
\hline & 11 & 4.29 & $(-2,8,50)$ & $A C C(L)$ \\
\hline & 10 & 3.70 & $(-12,60,-4)$ & OFC (L) \\
\hline \multirow[t]{2}{*}{ Healthy controls } & 69 & 4.00 & $(42,50,-4)$ & OFC (R) \\
\hline & 18 & 3.71 & $(0,28,24)$ & $\mathrm{ACC}(\mathrm{L})$ \\
\hline \multicolumn{5}{|l|}{ Neutral > Discordant } \\
\hline \multirow[t]{3}{*}{ Healthy controls } & 99 & 4.04 & $(32,46,20)$ & DPFC (R) \\
\hline & 16 & 3.48 & $(-50,34,6)$ & VLPFC (L) \\
\hline & 13 & 3.90 & $(42,48,0)$ & DPFC (R) \\
\hline IGT risky phase & $K_{E}$ & T & $(x, y, z)$ & ROI selected \\
\hline \multicolumn{5}{|l|}{ Discordant > Neutral } \\
\hline \multirow[t]{2}{*}{ Suicide attempters } & 39 & 4.31 & $(36,24,52)$ & DPFC (R) \\
\hline & 10 & 3.78 & $(-26,16,-16)$ & OFC (L) \\
\hline \multicolumn{5}{|l|}{ Neutral > Concordant } \\
\hline \multirow[t]{3}{*}{ Affective controls } & 41 & 4.12 & $(4,30,-4)$ & $A C C(R)$ \\
\hline & 27 & 3.84 & $(-34,44,20)$ & DPFC (L) \\
\hline & 14 & 3.61 & $(-24,56,2)$ & MPFC (L) \\
\hline
\end{tabular}

IGT lowa Gambling Task, ROI Regions of interest; (L) Left, (R) Right, VLPFC Ventrolateral prefrontal cortex, ACC Anterior cingulate cortex, OFC Orbitofrontal cortex, DPFC Dorsal prefrontal cortex, MPFC Medial prefrontal cortex.

$(p<0.001, k=39)$ and left OFC $(p<0.001, k=10)$ in patients with SA (Fig. 3b).

\section{Correlations between $\mathrm{FMRI}$ and behavioral data}

The ROI beta values of each patient for the ambiguous and risky phases were extracted. Pearson's correlations between the beta values and the behavioral data (reaction time and IG index) showed significant negative correlations between the IG index $(\mathrm{CD}-\mathrm{AB})$ during the risky phase and the activation of all the brain areas (all $p<0.05$ FDR corrected). Thus, higher activation in prefrontal areas was associated with lower preference for the safe decks (lower IG index) during the risky phase (see Table 1 in supplementary material).

\section{Discussion}

The present study showed that during a decisionmaking task that included emotional feedback, behavioral performance and brain activation patterns are different in euthymic suicide attempters compared with controls (AC and $\mathrm{HC}$ ).

We did not found significant effects of condition (congruent, incongruent or neutral feedback) at behavioral level. It is not on line with results from Aite et al. ${ }^{25}$ where, conducted, in healthy controls. Congruent feedback was associated with safety choices whereas incongruent feedback was associated with risky choices during IGT. This contradiction may be explained by several reasons. First, our sample included patients with history of suicide attempt, characterized by difficulties in decision making ${ }^{20}$. Second, the lack of power in healthy subjects may explain the lack of results for this sub-group. Third, while Aite et al. ${ }^{25}$ used fearful faces for the negative feedback, we used angry faces.

During the IGT ambiguous phase, the IG index was not different in the three groups, whereas concordant feedback was associated with slower reaction times in the SA group, as previously reported by Chen et $\mathrm{al}^{24}$. They showed that congruence between emotional and monetary outcomes lowered the reaction times during decision making ${ }^{24}$. Moreover, another study found that healthy women tend to take more risks after concordant feedback (monetary losses combined with angry faces) ${ }^{39}$.

Our neuroimaging results may suggest that suicide attempters did not correctly benefit from congruent emotional feedback during the IGT phase of ambiguous uncertainty. In both control groups, activation of OFC and $\mathrm{ACC}$ was reduced during risky vs. safe choices in the concordant vs. neutral feedback condition. Regulation of emotional conflicts (i.e., processing of task-relevant stimuli in conflict with incongruent emotional information) increases ACC activity ${ }^{40}$. A decrease in ACC suggests that congruent feedback facilitates emotional conflict resolution. In parallel, OFC implication decreased because congruent feedback might also have facilitated the attribution of value and risk during decision making ${ }^{17}$. Conversely, activity in these areas was not decreased in suicide attempters. This might suggest that differently from controls, suicide attempters did not process concordant feedback. On the other hand, VLPFC activation when receiving concordant feedback during the risky vs. safe choices was reduced only in suicide attempters, in agreement with a previous study showing a decrease in VLPFC activation in suicide attempters during risky vs. safe choices ${ }^{14}$.

During the IGT risky phase, the performance of suicide attempters in the discordant feedback condition was worse than that of healthy controls and also compared with their own results in the neutral and concordant 
A)

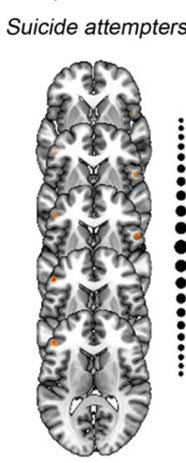

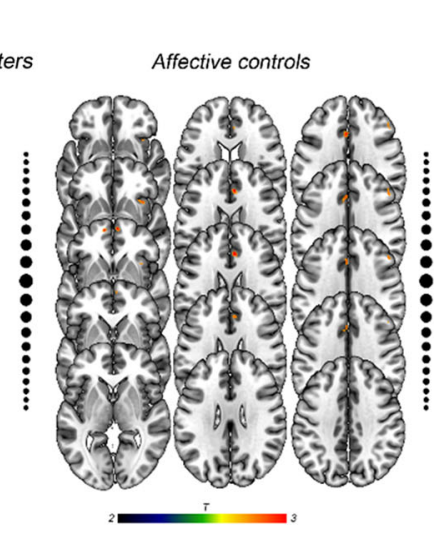

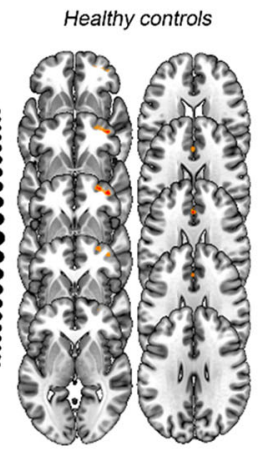

B)

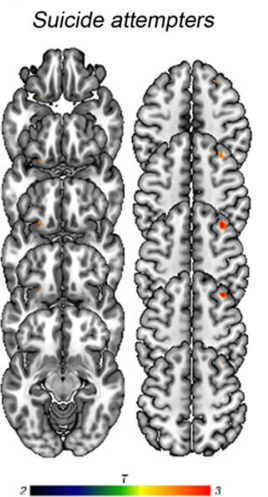

Fig. 3 Brain activation during risky ( $A$ and $B$ decks) vs. safe ( $C$ and $D$ decks) choices in the lowa Gambling Task. a Neutral $>$ Concordant emotional feedback condition for suicide attempters, affective controls and healthy controls during the ambiguous phase. $\mathbf{b}$ Discordant $>$ neutral emotional feedback condition for suicide attempters during the risky phase.

feedback conditions. This result might suggest altered performances under risky uncertainty with discordant feedback in suicide attempters. Discordant feedback may increase the probability of choosing risky decks in order to avoid angry faces that have higher salience in suicide attempters than healthy controls. At the neuroanatomical level, activation of OFC and DPFC was higher in suicide attempters for risky vs. safe choices in the discordant feedback compared with the neutral feedback condition. The incongruence between emotional feedback and choice (independently of the monetary feedback) may increase the executive control required to understand the decision-making task and to pick cards from the advantageous decks. Moreover, previous research using emotional images to create interference during a Stroop task showed that emotional negative information disturbed the executive control and increased OFC activation ${ }^{41}$. Suicide attempters are characterized by deficits in executive functions linked to the prefrontal areas, such as inhibitory control and working memory ${ }^{42,43}$. The increase in DPFC activation observed in suicide attempters but not in controls may reflect an impaired emotional-cognitive regulation due to incongruent emotional information ${ }^{20}$.

Our study has some limitations. First, the small size of the different groups increased the risk of type-I and typeII errors. This limits the application of stringent corrections ${ }^{44}$ and the adjustment for several variables. However, this was the first study on the interaction between emotional and monetary feedback in a clinical sample of suicide attempters. Second, ongoing pharmacological treatments also might have influenced brain activation in patients. However, medication load was comparable between patients' groups. Third, only women were included in the study. This did not allow assessing the gender effect and limits the generalization of our findings.
Nonetheless, our study proposes a new approach to investigate how socially relevant information might influence decision-making in suicide attempters. These results strengthen the evidence for a specific deficit in prefrontal cortex activity during decision making that may be part of the suicidal diathesis. Our behavioral results suggest that patients with suicidal vulnerability avoid negative social feedback, despite the expected outcome. It has been demonstrated that dysfunctional evaluation of the social context is an important marker of suicidal vulnerability ${ }^{27}$. Our study shows that in suicide attempters, both prefrontal brain activity and behavior are altered during decision making in the presence of social feedback. It suggests that emotional feedback does not have the same effect in suicide attempters and in affective and healthy controls. Consequently, suicide attempters may be more vulnerable to social stress ${ }^{45}$, increasing the risk of more deleterious decisions. Thus, patients with history of suicidal acts might benefit from therapies that focus on emotion visualization and interpretation to better adapt to the challenges of daily life.

\section{Acknowledgements}

We thank Dr E. Andermarcher for her careful reading of the manuscript. F.C. received a MSc grant from Servier Foundation. P.C. received a grant from the National Agency for Research ANR MNPS 2009 "VASCO".

\section{Author details \\ ${ }^{1}$ Department of Emergency Psychiatry and Acute Care, CHU Montpellier, Montpellier, France. ${ }^{2}$ PSNREC, Univ Montpellier, INSERM, CHU de Montpellier, Montpellier, France. ${ }^{3}$ Department of Neuroradiology, Academic hospital of Montpellier \& U1051, Institut of Neurosciences of Montpellier, Montpellier, France. ${ }^{4} \mathrm{I} 2 \mathrm{FH}$, Institut d'Imagerie Fonctionnelle Humaine, Montpellier University Hospital, Gui de Chauliac Hospital, Montpellier, France}

Conflict of interest

The authors declare that they have no conflict of interest. 


\section{Ethical standards}

The authors assert that all procedures contributing to this work comply with the ethical standards of the relevant national and institutional committees on human experimentation and with the Helsinki Declaration of 1975, as revised in 2008 .

\section{Publisher's note}

Springer Nature remains neutral with regard to jurisdictional claims in published maps and institutional affiliations.

Supplementary Information accompanies this paper at (https://doi.org/ 10.1038/s41398-020-00995-z)

Received: 17 January 2020 Revised: 25 August 2020 Accepted: 3 September 2020

Published online: 18 September 2020

\section{References}

1. Bergmans, Y., Gordon, E. \& Eynan, R. Surviving moment to moment: the experience of living in a state of ambivalence for those with recurrent suicide attempts. Psychol. Psychother. Theory Res. Pract. 90, 633-648 (2017).

2. Bechara, A., Damasio, A. R., Damasio, H. \& Anderson, S. W. Insensitivity to future consequences following damage to human prefrontal cortex. Cognition $\mathbf{5 0}$ 7-15 (1994).

3. Jollant, F. et al. Impaired decision making in suicide attempters. Am. J. Psychiatry 162, 304-310 (2005).

4. Jollant, F. et al. Impaired decision-making in suicide attempters may increase the risk of problems in affective relationships. J. Affect. Disord. 99, 59-62 (2007).

5. Richard-Devantoy, S. et al. Distinct alterations in value-based decision-making and cognitive control in suicide attempters: toward a dual neurocognitive model. J. Affect. Disord. 151, 1120-1124 (2013).

6. Moniz, M. et al. The influence of planning and response inhibition on cognitive functioning of non-psychotic unipolar depressed suicide attempters. Eur. J. Psychol. 13, 717-732 (2017).

7. Hegedús, K. M. et al. Decision-making performance of depressed patients within 72 h following a suicide attempt. J. Affect. Disord. 235, 583-588 (2018).

8. Richard-Devantoy, S., Olié, E., Guillaume, S. \& Courtet, P. Decision-making in unipolar or bipolar suicide attempters. J. Affect. Disord. 190, 128-136 (2016).

9. Jollant, F., Guillaume, S., Jaussent, I., Bechara, A. \& Courtet, P. When knowing what to do is not sufficient to make good decisions: deficient use of explicit understanding in remitted patients with histories of suicidal acts. Psychiatry Res. 210, 485-490 (2013).

10. Bridge, J. A. et al. Impaired decision making in adolescent suicide attempters. J. Am. Acad. Child Adolesc. Psychiatry, https://doi.org/10.1016/j.jaac.2012.01.002 (2012).

11. Bechara, a, Damasio, H., Tranel, D. \& Damasio, A. R. The lowa Gambling Task and the somatic marker hypothesis: some questions and answers. Trends Cogn. Sci. 9, 159-162 (2005).

12. Buelow, M. T., Okdie, B. M. \& Blaine, A. L. Seeing the forest through the trees: improving decision making on the lowa gambling task by shifting focus from short- to long-term outcomes. Front. Psychol. 4, 773 (2013).

13. Brand, M., Recknor, E. C., Grabenhorst, F. \& Bechara, A. Decisions under ambiguity and decisions under risk: correlations with executive functions and comparisons of two different gambling tasks with implicit and explicit rules. J. Clin. Exp. Neuropsychol. 29, 86-99 (2007)

14. Jollant, F. et al. Decreased activation of lateral orbitofrontal cortex during risky choices under uncertainty is associated with disadvantageous decisionmaking and suicidal behavior. Neuroimage, https://doi.org/10.1016/j. neuroimage.2010.03.027 (2010).

15. Olié, E. et al. Processing of decision-making and social threat in patients with history of suicidal attempt: a neuroimaging replication study. Psychiatry Res. Neuroimaging 234, 369-377 (2015).

16. Ding, Y. et al. Altered brain processing of decision-making in healthy firstdegree biological relatives of suicide completers. Mol. Psychiatry https:/doi. org/10.1038/mp.2016.221 (2016).

17. Grabenhorst, F. \& Rolls, E. T. Value, pleasure and choice in the ventral prefrontal cortex. Trends Cogn. Sci., https://doi.org/10.1016/j.tics.2010.12.004 (2011).
18. Kouneiher, F., Charron, S. \& Koechlin, E. Motivation and cognitive control in the human prefrontal cortex. Nat. Neurosci., https://doi.org/10.1038/nn.2321 (2009).

19. Dixon, M. L. \& Christoff, K. The lateral prefrontal cortex and complex valuebased learning and decision making. Neurosci. Biobehav. Rev., https://doi.org/ 10.1016/j.neubiorev.2014.04.011 (2014).

20. Jollant, F., Lawrence, N. L., Olié, E., Guillaume, S. \& Courtet, P. The suicidal mind and brain: a review of neuropsychological and neuroimaging studies. World J. Biol. Psychiatry 12, 319-339 (2011).

21. Damasio, A. R., Everitt, B. J. \& Bishop, D. The somatic marker hypothesis and the possible functions of the prefrontal cortex [and discussion]. Philos. Trans. R. Soc B Biol. Sci. 351, 1413-1420 (1996).

22. Averbeck, B. B. \& Duchaine, B. Integration of social and utilitarian factors in decision making. Emotion, https://doi.org/10.1037/a0016509 (2009).

23. Van Kleef, G. A., De Dreu, C. K. W. \& Manstead, A. S. R. The interpersonal effects of anger and happiness in negotiations. J. Pers. Soc. Psychol., https://doi.org/ 10.1037/0022-3514.86.1.57 (2004).

24. Chen, X., Zheng, T., Han, L., Chang, Y. \& Luo, Y. The neural dynamics underlying the interpersonal effects of emotional expression on decision making. Sci. Rep., https://doi.org/10.1038/srep46651 (2017).

25. Aïte, A. et al. Impact of emotional context congruency on decision making under ambiguity. Emotion, https://doi.org/10.1037/a0031345 (2013).

26. Jollant, F. et al. Orbitofrontal cortex response to angry faces in men with histories of suicide attempts. Am. J. Psychiatry 165, 740-748 (2008).

27. Olié, E. et al. The experience of social exclusion in women with a history of suicidal acts: a neuroimaging study. Sci. Rep. 7, 89 (2017).

28. Oldfield, R. C. The assessment and analysis of handedness: the Edinburgh inventory. Neuropsychologia, https://doi.org/10.1016/0028-3932(71)90067-4 (1971).

29. Hamilton, M. A rating scale for depression. J. Neurol. Neurosurg. Psychiatry 23, 56-62 (1960).

30. Young, R. C., Biggs, J. T., Ziegler, V. E. \& Meyer, D. A. A rating scale for mania: reliability, validity and sensitivity. Br. J. Psychiatry 133, 429-435 (1978).

31. Van Heeringen, K. \& Mann, J. J. The neurobiology of suicide. Lancet Psychiatry 1, 63-72 (2014).

32. Sheehan, D. V. et al. The Mini-International Neuropsychiatric Interview (M.I.N.I.): The development and validation of a structured diagnostic psychiatric interview for DSM-IV and ICD-10. in. J. Clin. Psychiatry 59, 22-33 (1998).

33. Beck, A. T. Beck Depression Inventory. Depression 2006, 2-4 (1961).

34. Spielberger, C. (ed) Manual for the State-Trait Anxiety Inventory (STAI). (Consult. Psychol. Press, Palo Alto, 1983).

35. Baylé, F. J. et al. Structure factorielle de la traduction française de l'échelle d'impulsivité de Barratt (BIS-10). [Factor structure of the French translation of theBarratt Impulsivity Scale (BIS-10).]. Can. J. Psychiatry / La Rev. Can. Psychiatr. 45, 156-165 (2000).

36. Mackinnon, A., Ritchie, K. \& Mulligan, R. The measurement properties of a French language adaptation of the National Adult Reading Test. Int. J. Methods Psychiatr. Res. 8, 27-38 (1999).

37. Bernstein, D. P. et al. Initial reliability and validity of a new retrospective measure of child abuse and neglect. Am. J. Psychiatry 151, 1132-1136 (1994).

38. Lawrence, N. S., Jollant, F., O'Daly, O., Zelaya, F. \& Phillips, M. L. Distinct roles of prefrontal cortical subregions in the iowa gambling task. Cereb. Cortex 19, 1134-1143 (2009).

39. Chen, X., Yuan, H., Zheng, T., Chang, Y. \& Luo, Y. Females are more sensitive to opponent's emotional feedback: evidence from event-related potentials. Front. Hum. Neurosci., https://doi.org/10.3389/fnhum.2018.00275 (2018).

40. Egner, T., Etkin, A., Gale, S. \& Hirsch, J. Dissociable neural systems resolve conflict from emotional versus nonemotional distracters. Cereb. Cortex, https:// doi.org/10.1093/cercor/bhm179 (2008).

41. Melcher, T., Born, C. \& Gruber, O. How negative affect influences neural control processes underlying the resolution of cognitive interference: an event-related fMRI study. Neurosci. Res., https://doi.org/10.1016/j.neures.2011.05.007 (2011).

42. Richard-Devantoy, S., Berlim, M. T. \& Jollant, F. Suicidal behaviour and memory: a systematic review and meta-analysis. World J. Biol. Psychiatry 16, 544-566 (2015).

43. Richard-Devantoy, S., Berlim, M. T. \& Jollant, F. A meta-analysis of neuropsychological markers of vulnerability to suicidal behavior in mood disorders. Psychol. Med. 44, 1663-1673 (2014).

44. Lieberman, M. D. \& Cunningham, W. A. Type I and Type II error concerns in fMRI research: re-balancing the scale. Soc. Cogn. Affect. Neurosci. 4, 423-428 (2009).

45. Courtet, P. \& Olié, E. Neurobiology of suicidal behavior and social stress. J. Neurosci. Res. 98, 579-580 (2020). 